\title{
Biofilm Formation Ability of Arcobacter-like and Campylobacter Strains under Different Conditions and on Food Processing Materials
}

\author{
David Šilha *, Sabina Sirotková, Karolína Švarcová (D), Leona Hofmeisterová, Květa Koryčanová \\ and Lucie Šilhová
}

check for updates

Citation: Šilha, D.; Sirotková, S.; Švarcová, K.; Hofmeisterová, L.; Koryčanová, K.; Šilhová, L. Biofilm Formation Ability of Arcobacter-like and Campylobacter Strains under Different Conditions and on Food Processing Materials. Microorganisms 2021, 9, 2017. https://doi.org/ 10.3390/microorganisms 9102017

Academic Editor:

Wolf-Rainer Abraham

Received: 20 August 2021

Accepted: 21 September 2021

Published: 23 September 2021

Publisher's Note: MDPI stays neutral with regard to jurisdictional claims in published maps and institutional affiliations.

Copyright: (c) 2021 by the authors. Licensee MDPI, Basel, Switzerland. This article is an open access article distributed under the terms and conditions of the Creative Commons Attribution (CC BY) license (https:// creativecommons.org/licenses/by/ $4.0 /)$.
Department of Biological and Biochemical Sciences, Faculty of Chemical Technology, University of Pardubice, Studentská 573, 53210 Pardubice, Czech Republic; toskan@centrum.cz (S.S.);

Karolina.Svarcova@student.upce.cz (K.Š.); Leona.Hofmeisterova@student.upce.cz (L.H.);

Kveta.Korycanova@upce.cz (K.K.); Lucie.Silhova@atlas.cz (L.Š.)

* Correspondence: David.Silha@upce.cz; Tel.: +420-466-037-765

\begin{abstract}
Campylobacter jejuni is the most frequent cause of bacterial gastrointestinal food-borne infection worldwide. The transmission of Campylobacter and Arcobacter-like species is often made possible by their ability to adhere to various abiotic surfaces. This study is focused on monitoring the biofilm ability of 69 strains of Campylobacter spp. and lesser described species of the Arcobacteraceae family isolated from food, water, and clinical samples within the Czech Republic. Biofilm formation was monitored and evaluated under an aerobic/microaerophilic atmosphere after cultivation for 24 or $72 \mathrm{~h}$ depending on the surface material. An overall higher adhesion ability was observed in arcobacters. A chi-squared test showed no association between the origin of the strains and biofilm activity $(p>0.05)$. Arcobacter-like species are able to form biofilms under microaerophilic and aerobic conditions; however, they prefer microaerophilic environments. Biofilm formation has already been demonstrated at refrigerator temperatures $\left(5^{\circ} \mathrm{C}\right)$. Arcobacters also showed higher biofilm formation ability at the temperature of $30^{\circ} \mathrm{C}$. This is in contrast to Campylobacter jejuni NP 2896, which showed higher biofilm formation ability at temperatures of $5-30^{\circ} \mathrm{C}$. Overall, the results demonstrated the biofilm formation ability of many strains, which poses a considerable risk to the food industry, medical practice, and human health.
\end{abstract}

Keywords: Arcobacter-like; Aliarcobacter spp.; Campylobacter spp.; biofilm formation; abiotic surfaces; temperature condition; food processing materials

\section{Introduction}

Biofilms are microbial communities growing on the surface or interface of materials. Cells are "interconnected" in a biofilm, usually via a matrix formed of extracellular polymeric substances (EPS) that they produce themselves [1]. The biofilm structure provides certain benefits to the microorganisms [2]. Bacteria living in biofilms exhibit increased resistance to host defense mechanisms and up to 1000 times higher natural resistance, e.g., to antibiotics $[3,4]$. The current trend is also to monitor the influence of many natural substances on the formation of microbial biofilms [5,6]. In nature, we often observe multispecies biofilms created on abiotic or biotic surfaces [7]. Biofilm formation requires a special type of signaling, known as quorum sensing (QS; also called density sensing) among the microorganism cells [8]. QS controls a variety of physiological behaviors of Gram-positive and Gram-negative bacteria and there are a variety of mechanisms and signal molecules used in QS to transmit information [9]. Biofilm formation comprises several stages, namely, the initial attachment to the surface, micro-colony formation, maturation, and detachment (dispersion) of biofilm [8]. The biofilm formation itself can be reduced, for example, by reducing the adhesion of microorganisms, or we must subsequently address the eradication 
of the biofilm formed [2]. However, biofilm formation is also largely dependent on various environmental influences and mechanisms affecting microbial cell gene expression [10]. Adhesion to the surfaces is a fundamental step in biofilm formation and leads to significant changes in cell metabolism, mainly to the expression of EPS and various microbial surface components, which recognize adhesive matrix molecules [11].

Biofilms formed on food processing surfaces are of great concern. The hygiene of these surfaces affects the quality of food products [12]. Biofilms constituted by pathogenic bacteria can result in product contamination that may lead to health problems. In fact, biofilms are known to be frequent sources of infections and many foodborne outbreaks have been associated with them $[13,14]$. A number of methods are used to demonstrate biofilm formation. The gold standard is the widely used Christensen phenotypic colorimetric method that uses crystal violet to color the biofilm layer. Genotypic methods that demonstrate the presence of genes responsible for biofilm formation are also useful [15-17].

It is estimated that over $80 \%$ of the bacterial population on Earth is capable of biofilm formation under certain conditions [18]. Biofilm-forming bacteria also include Arcobacter-like and Campylobacter strains $[17,19,20]$. These are Gram-negative bacteria that grow at $30-37{ }^{\circ} \mathrm{C}$, or even up to $42{ }^{\circ} \mathrm{C}$ in the case of campylobacters. The basic difference between arcobacters and the Campylobacter spp. is the ability of arcobacters to grow at lower temperatures and in both aerobic and microaerophilic environments. In many studies, the occurrence of these bacteria has been reported mainly in food and water, but also in the environment [21,22]. Recently, based on a wide comparative genomic analysis, reclassification and a new Arcobacteraceae family was proposed [23]. Further, the taxonomy of the genus Arcobacter has been revisited using the core genome of 57 strains, a multilocus sequence analysis with 13 housekeeping genes, and genomic indexes like average nucleotide identity (ANI), in silico DNA-DNA hybridization (isDDH), average amino acid identity (AAI), percentage of conserved proteins, etc. According to results, genomic and phylogenetic groups were delimited and six different genera, including Aliarcobacter gen. nov., were proposed. This genus comprises seven species, including Aliarcobacter butzleri comb. nov., Aliarcobacter cryaerophilus comb. nov., Aliarcobacter skirrowii comb. nov., etc. [24]. These microorganisms can cause gastroenteritis and other human and animal diseases. The association of arcobacters with human disease has been demonstrated for A. butzleri, A. cryaerophilus, A. skirrowii and A. thereius [25,26]. For these bacteria, survival in the form of a biofilm is essential, as they can thus better colonize hosts or contaminate food factory environments [22,27].

The aim of this study was to monitor the biofilm formation of many Arcobacter-like and Campylobacter strains depending on the conditions of the cultivation atmosphere, the cultivation time, and also on the type of abiotic surface in terms of bacterial cell adherence. For selected strains, biofilm formation was monitored depending on the temperature and type of material surface used in the industry. Collection strains were included in the study, some of which do not have sufficient evidence in the literature about their ability to form biofilms. However, many Arcobacter-like strains isolated within the Czech Republic from food and water samples were also included. Moreover, as far as we know, this study is one of the few that also includes clinical isolates of both Campylobacter and Arcobacter-like species for biofilm formation testing.

\section{Materials and Methods}

\subsection{Bacterial Strains and Identification by $m-P C R$ and $16 S$ RNA-RFLP}

A total of 69 isolates and collection strains of the Arcobacter-like bacteria and Campylobacter spp. were used to determine their biofilm formation on different surfaces and under different culture conditions. Bacterial strains were used from the Czech Collection of Microorganisms (CCM), Culture Collection University of Göteborg (CCUG), Belgian Co-ordinated Collections of Microorganisms (LMG), or strains isolated at the University of Pardubice (UPa) according to a previously described protocol [21]. Furthermore, some clinical strains were obtained from Pardubice Region Hospital, a.s. (Litomyšl Hospital, 
Pardubice Hospital) and Náchod Regional Hospital, a.s. (Náchod Hospital). Before testing, cultures were grown on tryptone soya agar (TSA; HiMedia, Mumbai, India) either for $48 \mathrm{~h}$ at $30^{\circ} \mathrm{C}$ under aerobic conditions (Arcobacter-like strains) or for $48 \mathrm{~h}$ at $42{ }^{\circ} \mathrm{C}$ under microaerophilic conditions (Campylobacter spp.). Cells were harvested and suspended in physiological saline to a $0.5 \mathrm{McF}$ arland scale (ca $1.5 \times 10^{8} \mathrm{CFU} / \mathrm{mL}$ ). The suspension of cells was then diluted to an appropriate density before testing. For the verification of the exact number of cells in the prepared cell suspension, a presumptive density of $10^{3} \mathrm{CFU} / \mathrm{mL}$ was counted on TSA.

All strains were identified using the m-PCR method [28]. Briefly, PCR reactions were performed in a reaction mixture (50 $\mu \mathrm{L}$ final volume) composed of PCR water, $5 \mu \mathrm{L}$ of $10 \times$ PCR buffer, $1.5 \mathrm{U}$ Taq polymerase and a deoxynucleotide triphosphate mixture at a final concentration of $0.2 \mathrm{mM}$ each. The mixture also contained $1.5 \mathrm{mmol}$ of $\mathrm{MgCl}_{2}$ and $50 \mathrm{pmol}$ of each primer (ButR, SkiR, TherR, CibR, ArcoF, GyrasF, and GyrasR). Prior to cycling, samples were heated to $94^{\circ} \mathrm{C}$ for $3 \mathrm{~min}$. The PCR assay involved 30 cycles of denaturation $\left(94{ }^{\circ} \mathrm{C}, 45 \mathrm{~s}\right)$, primer annealing $\left(58^{\circ} \mathrm{C}, 45 \mathrm{~s}\right)$, and chain extension $\left(72{ }^{\circ} \mathrm{C}, 2 \mathrm{~min}\right)$ with a final elongation step at $72{ }^{\circ} \mathrm{C}$ for $5 \mathrm{~min}$. PCR products were separated in $2 \%$ agarose gel in $0.5 \times$ TBE buffer at $120 \mathrm{~V}$ for $2 \mathrm{~h}$. Gels were stained with ethidium bromide $(1 \mu \mathrm{g} / \mathrm{mL})$.

Due to the possibility of distinguishing A. cryaerophilus strains into $1 \mathrm{~A}$ and $1 \mathrm{~B}$ and also to increase the reliability of identification based on previous knowledge, all strains were subjected to identification according to the 16S rRNA-RFLP protocol [29]. Genomic DNA was used as a template for the PCR amplification of a $1026 \mathrm{bp}$ region of the $16 \mathrm{~S}$ rRNA gene, as previously described [30]. Amplicons were digested with MseI or MnII/BfaI (BioLabs New England, Ipswich, MA, USA) in a $25 \mu \mathrm{L}$ final volume consisting of $0.5 \mu \mathrm{L}$ of the PCR amplicons, $5 \mathrm{U}$ of the enzyme (BioLabs New England, Ipswich, MA, USA), $2.5 \mu \mathrm{L}$ of $10 \times$ CutSmart buffer (BioLabs New England, Ipswich, MA, USA), and PCR water. The reaction mixture was digested at $37^{\circ} \mathrm{C}$ for $15 \mathrm{~min}(\mathrm{MseI}$ or $M n \mathrm{II})$ or $1 \mathrm{~h}(\mathrm{BfaI})$. Enzyme activity was inhibited at $65{ }^{\circ} \mathrm{C}$ for $20 \mathrm{~min}$ (MseI, MnII) or at $80^{\circ} \mathrm{C}$ for $20 \mathrm{~min}$ (BfaI) in accordance with the manufacturer's instructions. The digested products were separated on $3 \%$ agarose gels in $1 \times$ TBE buffer at $100 \mathrm{~V}$ for $120 \mathrm{~min}$. A $50 \mathrm{bp}$ DNA size marker (BiotechRabbit, Berlin, Germany) was used to confirm the expected amplicon size of each target gene. Gels were stained with ethidium bromide $(1 \mu \mathrm{g} / \mathrm{mL})$ and later photographed on a UV transilluminator.

\subsection{Determination of Biofilm Formation in Microtiter Plate at Various Conditions}

Biofilm formation was monitored in flat-bottomed microtiter plates (SPL Life Sciences, Pocheon-si, South Korea) as previously described [31,32] with some modifications. Briefly, $100 \mu \mathrm{L}$ of cell suspension $\left(10^{7} \mathrm{CFU} / \mathrm{mL}\right.$ ) in brain heart infusion broth (BHI; Himedia, Mumbai, India) was inoculated into 96-well flat-bottomed microtiter polystyrene plates (SPL Live Sciences Co., Ltd., Korea). After incubation under specific conditions, the wells were rinsed thoroughly five times with sterile distilled water and dried. Biofilm fixation was performed with $2 \%$ sodium acetate $(15 \mathrm{~min}$ ) and biofilm-forming cells were stained with $100 \mu \mathrm{L}$ of $1 \%$ crystal violet (Sigma-Aldrich, St. Louis, MO, USA). After 15 min of staining, the plate was repeatedly washed and dried. Thereafter, the biofilm-associated violet was solubilized with $96 \%$ ethanol and the absorbance (OD) of the solution was measured in a new plate at $595 \mathrm{~nm}$ (Infinite M200, Tecan, Männedorf, Switzerland). There were 24 wells in each experiment, and the experiments were independently repeated three times.

The biofilm formation level of the Arcobacter-like strains was categorized according to the classification system previously described [32] as non-adherent $\left(\mathrm{N} ; \mathrm{OD} \leq \mathrm{OD}_{\mathrm{C}}\right)$ or weakly (W; $\left.\mathrm{OD}_{\mathrm{C}}<\mathrm{OD} \leq 2 \times \mathrm{OD}_{\mathrm{C}}\right)$, moderately $\left(\mathrm{M} ; 2 \times \mathrm{OD}_{\mathrm{C}}<\mathrm{OD} \leq 4 \times \mathrm{OD}_{\mathrm{C}}\right)$, or strongly adherent $\left(\mathrm{S} ; 4 \times \mathrm{OD}_{\mathrm{C}}<\mathrm{OD}\right.$ ), where $\mathrm{OD}_{\mathrm{C}}$ (cut-off $\mathrm{OD}$ ) is defined as three standard deviations above the mean OD of the negative control (blank value). The measured and calculated $\mathrm{OD} / \mathrm{OD}_{\mathrm{C}}(0.111 / 0.120)$ values were the same for all measurements. 


\subsection{Determination of Biofilm Formation in Glass Tube at Various Conditions}

Biofilm formation was monitored in glass tubes as previously described by [15] with some modifications. Briefly, $2 \mathrm{~mL}$ of cell suspension $\left(10^{7} \mathrm{CFU} / \mathrm{mL}\right)$ in brain heart infusion broth (BHI; Himedia, Mumbai, India) was inoculated into sterile borosilicate glass tubes. After incubation under specific conditions, the tubes were rinsed thoroughly five times with sterile distilled water and dried. The tubes were stained with $1 \%$ crystal violet and then incubated at room temperature for $15 \mathrm{~min}$. The tubes were washed three times with distilled water and dried completely at $37^{\circ} \mathrm{C}$.

Evaluation of biofilm formation was performed visually by comparing with a weakly adherent E. coli CCM 3954 and a strongly adherent S. aureus CCM 4224. All experiments were repeated at least in triplicate and independently repeated three times. The results are recorded as non-adherent $(\mathrm{N})$, weakly $(\mathrm{W})$, moderately $(\mathrm{M})$, or strongly $(\mathrm{S})$ adherent.

\subsection{Biofilm Formation of Selected Adherent Strains on Food Processing Materials}

Biofilm formation on food processing materials was monitored as previously described with some modifications [33]. Biofilm formation ability of selected strains (A. defluvii LMG 25694, A. butzleri UPa 2013/3, A. cryaerophilus 1B UPa 2012/1, C. jejuni NP 2896) was evaluated after incubation in different liquid environments at $5,10,25,30$, and $42{ }^{\circ} \mathrm{C}$ for $24 \mathrm{~h}$ under aerobic atmosphere. Materials intended for food the industry or kitchen food preparation were chosen for further testing - stainless steel (type 304 finish 2b; Terapol, Prague, Czech Republic), plastic (PE-LD, plastic cutting board; IKEA, Sweden), and glass (microscope slides; Corning Glass Works, Corning, NY, USA). Material coupons $(4 \mathrm{~cm} \times 1 \mathrm{~cm})$ were first cleaned using an ultra-fine brush and liquid detergent. Coupons were rinsed in distilled water before being soaked in $70 \%$ ethanol. The coupons were allowed to air dry and then autoclaved before use. The biofilm sample was prepared by culturing $1 \mathrm{~mL}$ of bacterial suspension at a cell density of $10^{8} \mathrm{CFU} / \mathrm{mL}$ in $9 \mathrm{~mL}$ of tryptone soya broth (TSB; Himedie, Mumbai, India), BHI broth, or peptone water with $0.9 \% \mathrm{NaCl}$. After incubation, the coupon was removed from the culture medium and rinsed gently but repeatedly with sterile distilled water to remove planktonic cells. Biofilm cells were scraped off with a cotton swab, which was then transferred to a tube with $10 \mathrm{~mL}$ PBS. After intensive shaking of the swab (vortex, $2 \mathrm{~min}$ ) and sonication $(48 \mathrm{kHz}, 3 \mathrm{~min}$ ), the amount of biofilm-forming cells was monitored by culture. All experiments were repeated in at least triplicate.

\subsection{Statistical Analysis}

Statistical analysis was performed with Excel 2010 (Microsoft, Redmond, WA, USA) and Statistica 12 (StatSoft, Tulsa, OK, USA). Extreme values were tested with the DeanDixon test, and all remoteness values were excluded with $95 \%$ probability. Median and standard deviation were determined from the remaining values. A possible source of error resulting from insufficient dye washing resulting in an increase in absorbance was also considered, and absorbance values that were too high compared to other measured values were excluded.

Statistical significance was determined by chi-squared test. A $p$ value of $<0.05$ was considered to be statistically significant. The calculated standard deviations of the measurements are not shown in Tables $1-4$, but these values did not exceed $5 \%$ in any experiment. 
Table 1. Biofilm formation of A. butzleri strains on plastic/glass material at $30^{\circ} \mathrm{C}$.

\begin{tabular}{|c|c|c|c|c|}
\hline \multirow{2}{*}{ Strains } & \multicolumn{2}{|c|}{ Exposure for $24 \mathrm{~h}$} & \multicolumn{2}{|c|}{ Exposure for $72 \mathrm{~h}$} \\
\hline & Aerobic & Microaerophilic & Aerobic & Microaerophilic \\
\hline A. butzleri UPa $2012 / 3^{A}$ & $\mathrm{~W}(0.124) / \mathrm{M}$ & $\mathrm{W}(0.123) / \mathrm{W}$ & $\mathrm{W}(0.156) / \mathrm{M}$ & $\mathrm{W}(0.125) / \mathrm{W}$ \\
\hline A. butzleri UPa $2013 / 3^{B}$ & $\mathrm{~W}(0.173) / \mathrm{M}$ & $\mathrm{M}(0.262) / \mathrm{M}$ & $\mathrm{W}(0.129) / \mathrm{M}$ & $\mathrm{W}(0.136) / \mathrm{N}$ \\
\hline A. butzleri UPa $2013 / 10^{A}$ & $\mathrm{~N}(0.112) / \mathrm{W}$ & $\mathrm{N}(0.116) / \mathrm{W}$ & $\mathrm{N}(0.113) / \mathrm{M}$ & $\mathrm{W}(0.125) / \mathrm{M}$ \\
\hline A. butzleri UPa $2013 / 15^{A}$ & $\mathrm{~N}(0.116) / \mathrm{M}$ & $\mathrm{W}(0.121) / \mathrm{N}$ & $\mathrm{W}(0.140) / \mathrm{M}$ & $\mathrm{W}(0.142) / \mathrm{W}$ \\
\hline A. butzleri UPa $2013 / 30^{B}$ & $\mathrm{~W}(0.149) / \mathrm{M}$ & $\mathrm{W}(0.178) / \mathrm{M}$ & $\mathrm{W}(0.124) / \mathrm{W}$ & $\mathrm{W}(0.137) / \mathrm{M}$ \\
\hline A. butzleri UPa $2013 / 31^{B}$ & $\mathrm{~W}(0.121) / \mathrm{M}$ & $\mathrm{W}(0.126) / \mathrm{M}$ & $\mathrm{W}(0.126) / \mathrm{W}$ & $\mathrm{W}(0.132) / \mathrm{W}$ \\
\hline A. butzleri UPa $2013 / 32^{B}$ & $\mathrm{~W}(0.141) / \mathrm{W}$ & $\mathrm{W}(0.139) / \mathrm{N}$ & $\mathrm{W}(0.123) / \mathrm{W}$ & $\mathrm{W}(0.123) / \mathrm{W}$ \\
\hline A. butzleri UPa $2013 / 33^{B}$ & $\mathrm{~N}(0.112) / \mathrm{W}$ & $\mathrm{N}(0.117) / \mathrm{W}$ & $\mathrm{W}(0.122) / \mathrm{N}$ & $\mathrm{W}(0.127) / \mathrm{N}$ \\
\hline A. butzleri UPa $2013 / 36^{A}$ & $\mathrm{~W}(0.121) / \mathrm{W}$ & $\mathrm{W}(0.130) / \mathrm{W}$ & $\mathrm{N}(0.120) / \mathrm{M}$ & $W(0.129) / W$ \\
\hline A. butzleri UPa $2013 / 37^{B}$ & $\mathrm{~W}(0.181) / \mathrm{M}$ & $\mathrm{W}(0.207) / \mathrm{M}$ & $\mathrm{W}(0.124) / \mathrm{M}$ & $\mathrm{W}(0.135) / \mathrm{M}$ \\
\hline A. butzleri UPa $2014 / 51^{B}$ & $\mathrm{~W}(0.123) / \mathrm{W}$ & $\mathrm{W}(0.122) / \mathrm{N}$ & $\mathrm{W}(0.125) / \mathrm{M}$ & $\mathrm{W}(0.122) / \mathrm{W}$ \\
\hline A. butzleri UPa $2014 / 54^{B}$ & $\mathrm{~N}(0.117) / \mathrm{M}$ & $\mathrm{N}(0.116) / \mathrm{W}$ & $\mathrm{W}(0.129) / \mathrm{M}$ & $\mathrm{W}(0.124) / \mathrm{W}$ \\
\hline A. butzleri UPa $2015 / 1^{B}$ & $\mathrm{~W}(0.129) / \mathrm{M}$ & $\mathrm{W}(0.132) / \mathrm{W}$ & $\mathrm{N}(0.118) / \mathrm{M}$ & $W(0.122) / W$ \\
\hline A. butzleri UPa $2015 / 5^{A}$ & $\mathrm{~W}(0.152) / \mathrm{M}$ & $\mathrm{W}(0.202) / \mathrm{W}$ & $\mathrm{N}(0.118) / \mathrm{M}$ & $\mathrm{W}(0.121) / \mathrm{W}$ \\
\hline A. butzleri UPa $2015 / 6^{B}$ & $\mathrm{~W}(0.125) / \mathrm{N}$ & $\mathrm{W}(0.131) / \mathrm{N}$ & $\mathrm{N}(0.117) / \mathrm{W}$ & $\mathrm{W}(0.125) / \mathrm{N}$ \\
\hline A. butzleri UPa $2015 / 7^{B}$ & $\mathrm{M}(0.241) / \mathrm{M}$ & $\mathrm{W}(0.170) / \mathrm{W}$ & $\mathrm{W}(0.120) / \mathrm{M}$ & $\mathrm{W}(0.126) / \mathrm{W}$ \\
\hline A. butzleri UPa 2015/9 ${ }^{B}$ & $\mathrm{~W}(0.127) / \mathrm{W}$ & $\mathrm{W}(0.127) / \mathrm{W}$ & $\mathrm{N}(0.120) / \mathrm{W}$ & $\mathrm{W}(0.129) / \mathrm{W}$ \\
\hline A. butzleri UPa $2015 / 10^{B}$ & $\mathrm{~W}(0.141) / \mathrm{W}$ & $\mathrm{W}(0.140) / \mathrm{W}$ & $\mathrm{W}(0.137) / \mathrm{M}$ & $W(0.138) / W$ \\
\hline A. butzleri UPa $2015 / 11^{B}$ & $\mathrm{~W}(0.134) / \mathrm{N}$ & $\mathrm{W}(0.137) / \mathrm{N}$ & $\mathrm{W}(0.176) / \mathrm{M}$ & $\mathrm{W}(0.216) / \mathrm{W}$ \\
\hline A. butzleri UPa $2015 / 12^{B}$ & $\mathrm{~W}(0.176) / \mathrm{N}$ & $\mathrm{W}(0.135) / \mathrm{N}$ & $\mathrm{N}(0.117) / \mathrm{N}$ & $\mathrm{W}(0.122) / \mathrm{N}$ \\
\hline A. butzleri UPa $2015 / 13^{B}$ & $\mathrm{~N}(0.120) / \mathrm{N}$ & $\mathrm{W}(0.138) / \mathrm{N}$ & $\mathrm{W}(0.139) / \mathrm{M}$ & $\mathrm{W}(0.146) / \mathrm{N}$ \\
\hline A. butzleri UPa $2015 / 14^{B}$ & $\mathrm{~W}(0.130) / \mathrm{W}$ & $\mathrm{W}(0.136) / \mathrm{M}$ & $\mathrm{W}(0.124) / \mathrm{M}$ & $\mathrm{W}(0.136) / \mathrm{N}$ \\
\hline A. butzleri UPa $2015 / 15^{B}$ & $\mathrm{~W}(0.177) / \mathrm{N}$ & $\mathrm{W}(0.213) / \mathrm{N}$ & $\mathrm{W}(0.142) / \mathrm{M}$ & $\mathrm{W}(0.127) / \mathrm{N}$ \\
\hline A. butzleri UPa $2015 / 16^{B}$ & $\mathrm{~W}(0.198) / \mathrm{M}$ & $\mathrm{M}(0.375) / \mathrm{W}$ & $\mathrm{W}(0.147) / \mathrm{M}$ & $\mathrm{W}(0.217) / \mathrm{M}$ \\
\hline A. butzleri UPa $2015 / 18^{B}$ & $\mathrm{~W}(0.131) / \mathrm{M}$ & $\mathrm{W}(0.196) / \mathrm{W}$ & $\mathrm{N}(0.120) / \mathrm{N}$ & $\mathrm{W}(0.127) / \mathrm{W}$ \\
\hline A. butzleri UPa $2015 / 19^{B}$ & $\mathrm{~W}(0.133) / \mathrm{N}$ & $\mathrm{W}(0.134) / \mathrm{N}$ & $\mathrm{W}(0.132) / \mathrm{M}$ & $\mathrm{W}(0.149) / \mathrm{N}$ \\
\hline A. butzleri UPa $2015 / 20^{C}$ & $\mathrm{~W}(0.139) / \mathrm{N}$ & $\mathrm{W}(0.139) / \mathrm{N}$ & $\mathrm{W}(0.128) / \mathrm{M}$ & $\mathrm{W}(0.143) / \mathrm{N}$ \\
\hline A. butzleri UPa $2015 / 25^{B}$ & $\mathrm{M}(0.301) / \mathrm{W}$ & $\mathrm{W}(0.160) / \mathrm{N}$ & $\mathrm{W}(0.151) / \mathrm{M}$ & $\mathrm{W}(0.146) / \mathrm{W}$ \\
\hline A. butzleri $\mathrm{UPa} \mathrm{KK}^{B}$ & $\mathrm{~N}(0.109) / \mathrm{M}$ & $\mathrm{N}(0.117) / \mathrm{W}$ & $\mathrm{N}(0.119) / \mathrm{M}$ & $\mathrm{W}(0.121) / \mathrm{M}$ \\
\hline A. butzleri UPa $24 \mathrm{~A}^{B}$ & $\mathrm{~W}(0.138) / \mathrm{M}$ & $\mathrm{M}(0.418) / \mathrm{M}$ & $\mathrm{W}(0.169) / \mathrm{M}$ & $\mathrm{M}(0.246) / \mathrm{M}$ \\
\hline A. butzleri $\mathrm{UPa} 30 \mathrm{~B}^{B}$ & $\mathrm{~W}(0.131) / \mathrm{W}$ & $\mathrm{N}(0.118) / \mathrm{N}$ & $\mathrm{W}(0.137) / \mathrm{M}$ & $\mathrm{N}(0.118) / \mathrm{W}$ \\
\hline A. butzleri UPa $39-3^{C}$ & $\mathrm{~W}(0.143) / \mathrm{N}$ & $\mathrm{W}(0.134) / \mathrm{M}$ & $\mathrm{M}(0.242) / \mathrm{N}$ & $\mathrm{W}(0.164) / \mathrm{N}$ \\
\hline A. butzleri $\mathrm{UPa} 49 \mathrm{~B}^{B}$ & $\mathrm{~W}(0.130) / \mathrm{W}$ & $\mathrm{W}(0.128) / \mathrm{N}$ & $\mathrm{W}(0.124) / \mathrm{M}$ & $\mathrm{W}(0.122) / \mathrm{W}$ \\
\hline A. butzleri UPa $65 \mathrm{~A}^{B}$ & $\mathrm{~W}(0.130) / \mathrm{W}$ & $\mathrm{W}(0.128) / \mathrm{W}$ & $\mathrm{W}(0.128) / \mathrm{M}$ & $\mathrm{W}(0.173) / \mathrm{M}$ \\
\hline A. butzleri $\mathrm{UPa} 132 \mathrm{~A}^{B}$ & $\mathrm{~W}(0.127) / \mathrm{W}$ & $\mathrm{W}(0.151) / \mathrm{W}$ & $\mathrm{N}(0.113) / \mathrm{M}$ & $\mathrm{W}(0.125) / \mathrm{M}$ \\
\hline A. butzleri UPa $138 \mathrm{~A}^{B}$ & $\mathrm{~W}(0.141) / \mathrm{M}$ & $\mathrm{M}(0.265) / \mathrm{W}$ & $\mathrm{W}(0.122) / \mathrm{M}$ & $\mathrm{W}(0.153) / \mathrm{W}$ \\
\hline A. butzleri $\mathrm{UPa} 141 \mathrm{~B}^{B}$ & $\mathrm{~W}(0.123) / \mathrm{W}$ & $\mathrm{W}(0.137) / \mathrm{W}$ & $\mathrm{N}(0.116) / \mathrm{M}$ & $\mathrm{W}(0.134) / \mathrm{M}$ \\
\hline
\end{tabular}

${ }^{A}$ strain isolated from water; ${ }^{B}$ strain isolated from food, ${ }^{C}$ clinical isolate; UPa—strains isolated at University of Pardubice. N-nonadherent, $\mathrm{W}$-weakly adherent, $\mathrm{M}$-moderately adherent, S—-strongly adherent. Value in parentheses represents the actual measured absorbance value.

Table 2. Biofilm formation of $A$. cryaerophilus strains (subgroups $1 \mathrm{~A}$ and $1 \mathrm{~B}$ ) on plastic/glass material at $30^{\circ} \mathrm{C}$.

\begin{tabular}{|c|c|c|c|c|}
\hline \multirow{2}{*}{ Strains } & \multicolumn{2}{|c|}{ Exposure for $24 \mathrm{~h}$} & \multicolumn{2}{|c|}{ Exposure for $72 \mathrm{~h}$} \\
\hline & Aerobic & Microaerophilic & Aerobic & Microaerophilic \\
\hline A. cryaerophilus 1B UPa 2012/1 ${ }^{A}$ & $\mathrm{~W}(0.130) / \mathrm{M}$ & $\mathrm{W}(0.121) / \mathrm{W}$ & $\mathrm{W}(0.126) / \mathrm{M}$ & $\mathrm{W}(0.129) / \mathrm{W}$ \\
\hline A. cryaerophilus 1B UPa 2013/1 ${ }^{B}$ & $\mathrm{~N}(0.119) / \mathrm{N}$ & $\mathrm{W}(0.127) / \mathrm{W}$ & $\mathrm{W}(0.150) / \mathrm{M}$ & $\mathrm{W}(0.143) / \mathrm{M}$ \\
\hline A. cryaerophilus 1 A UPa $2013 / 12^{A}$ & $\mathrm{~N}(0.119) / \mathrm{N}$ & $\mathrm{W}(0.129) / \mathrm{N}$ & $\mathrm{W}(0.146) / \mathrm{W}$ & $\mathrm{W}(0.154) / \mathrm{W}$ \\
\hline A. cryaerophilus 1B UPa $2013 / 13^{A}$ & $\mathrm{~W}(0.128) / \mathrm{M}$ & $\mathrm{W}(0.165) / \mathrm{M}$ & $\mathrm{W}(0.124) / \mathrm{W}$ & $\mathrm{W}(0.134) / \mathrm{W}$ \\
\hline A. cryaerophilus 1B UPa $2013 / 14^{A}$ & $\mathrm{~W}(0.136) / \mathrm{N}$ & $\mathrm{W}(0.144) / \mathrm{W}$ & $\mathrm{W}(0.133) / \mathrm{N}$ & $\mathrm{W}(0.136) / \mathrm{N}$ \\
\hline A. cryaerophilus 1B UPa $2013 / 16^{A}$ & $\mathrm{~W}(0.121) / \mathrm{M}$ & $\mathrm{W}(0.123) / \mathrm{W}$ & $\mathrm{W}(0.124) / \mathrm{W}$ & $\mathrm{W}(0.129) / \mathrm{M}$ \\
\hline
\end{tabular}


Table 2. Cont.

\begin{tabular}{|c|c|c|c|c|}
\hline \multirow{2}{*}{ Strains } & \multicolumn{2}{|c|}{ Exposure for $24 \mathrm{~h}$} & \multicolumn{2}{|c|}{ Exposure for $72 \mathrm{~h}$} \\
\hline & Aerobic & Microaerophilic & Aerobic & Microaerophilic \\
\hline A. cryaerophilus 1B UPa 2013/17 ${ }^{B}$ & $\mathrm{~N}(0.118) / \mathrm{M}$ & $\mathrm{N}(0.117) / \mathrm{W}$ & $\mathrm{W}(0.127) / \mathrm{W}$ & $\mathrm{W}(0.129) / \mathrm{M}$ \\
\hline A. cryaerophilus 1B UPa $2013 / 28^{B}$ & $\mathrm{~N}(0.117) / \mathrm{M}$ & $\mathrm{W}(0.122) / \mathrm{W}$ & $\mathrm{W}(0.133) / \mathrm{M}$ & $\mathrm{W}(0.137) / \mathrm{W}$ \\
\hline A. cryaerophilus 1B UPa 2013/35 A & $\mathrm{W}(0.130) / \mathrm{M}$ & $\mathrm{W}(0.195) / \mathrm{M}$ & $\mathrm{N}(0.118) / \mathrm{M}$ & $\mathrm{N}(0.118) / \mathrm{W}$ \\
\hline A. cryaerophilus 1B UPa 2014/58 ${ }^{B}$ & $\mathrm{~W}(0.129) / \mathrm{W}$ & $\mathrm{W}(0.122) / \mathrm{W}$ & $\mathrm{W}(0.142) / \mathrm{M}$ & $\mathrm{W}(0.145) / \mathrm{W}$ \\
\hline A. cryaerophilus 1B UPa $2014 / 58 \mathrm{~A}^{B}$ & $\mathrm{~W}(0.128) / \mathrm{W}$ & $\mathrm{W}(0.137) / \mathrm{W}$ & $\mathrm{W}(0.155) / \mathrm{M}$ & $\mathrm{M}(0.242) / \mathrm{M}$ \\
\hline A. cryaerophilus 1B UPa 2014/58D ${ }^{B}$ & $\mathrm{~W}(0.144) / \mathrm{N}$ & $\mathrm{W}(0.137) / \mathrm{N}$ & $\mathrm{W}(0.151) / \mathrm{M}$ & $\mathrm{W}(0.177) / \mathrm{W}$ \\
\hline A. cryaerophilus 1B UPa 2014/59 ${ }^{B}$ & $\mathrm{~W}(0.127) / \mathrm{N}$ & $\mathrm{W}(0.132) / \mathrm{M}$ & $\mathrm{W}(0.147) / \mathrm{W}$ & $\mathrm{W}(0.135) / \mathrm{N}$ \\
\hline A. cryaerophilus $1 \mathrm{~B}$ UPa $130^{B}$ & $\mathrm{~W}(0.125) / \mathrm{W}$ & $\mathrm{W}(0.131) / \mathrm{N}$ & $\mathrm{W}(0.121) / \mathrm{S}$ & $\mathrm{N}(0.116) / \mathrm{M}$ \\
\hline
\end{tabular}

${ }^{A}$ strain isolated from water; ${ }^{B}$ strain isolated from food; UPa—strains isolated at University of Pardubice, N—non-adherent, W—-weakly adherent, $\mathrm{M}-$ moderately adherent, $\mathrm{S}$-strongly adherent. Value in parentheses represents the actual measured absorbance value.

Table 3. Biofilm formation of Arcobacter-reference strains on plastic/glass material at $30{ }^{\circ} \mathrm{C}$.

\begin{tabular}{ccccc}
\hline \multirow{2}{*}{ Strains } & \multicolumn{2}{c}{ Exposure for 24 $\mathbf{h}$} & \multicolumn{2}{c}{ Exposure for 72 h } \\
\cline { 2 - 5 } & Aerobic & Microaerophilic & Aerobic & Microaerophilic \\
\hline A. butzleri CCUG 30484 & $\mathrm{W}(0.152) / \mathrm{M}$ & $\mathrm{W}(0.179) / \mathrm{W}$ & $\mathrm{W}(0.178) / \mathrm{M}$ & $\mathrm{M}(0.241) / \mathrm{W}$ \\
A. butzleri LMG 10828 & $\mathrm{W}(0.126) / \mathrm{M}$ & $\mathrm{W}(0.130) / \mathrm{W}$ & $\mathrm{W}(0.132) / \mathrm{M}$ & $\mathrm{W}(0.178) / \mathrm{W}$ \\
A. cryaerophilus 1B CCM 3933 & $\mathrm{N}(0.118) / \mathrm{N}$ & $\mathrm{N}(0.114) / \mathrm{M}$ & $\mathrm{N}(0.120) / \mathrm{W}$ & $\mathrm{W}(0.195) / \mathrm{N}$ \\
A. cryaerophilus 1B CCM 7050 & $\mathrm{W}(0.133) / \mathrm{M}$ & $\mathrm{W}(0.138) / \mathrm{M}$ & $\mathrm{N}(0.116) / \mathrm{W}$ & $\mathrm{W}(0.130) / \mathrm{W}$ \\
A. defluvii LMG 25694 & $\mathrm{W}(0.228) / \mathrm{S}$ & $\mathrm{M}(0.379) / \mathrm{S}$ & $\mathrm{M}(0.414) / \mathrm{S}$ & $\mathrm{S}(0.504) / \mathrm{S}$ \\
A. lanthieri LMG 28517 & $\mathrm{W}(0.125) / \mathrm{W}$ & $\mathrm{W}(0.132) / \mathrm{N}$ & $\mathrm{W}(0.123) / \mathrm{M}$ & $\mathrm{W}(0.140) / \mathrm{N}$ \\
A. skirrovii LMG 6621 & $\mathrm{W}(0.126) / \mathrm{N}$ & $\mathrm{W}(0.130) / \mathrm{N}$ & $\mathrm{W}(0.136) / \mathrm{M}$ & $\mathrm{W}(0.129) / \mathrm{M}$ \\
A. thereius LMG 24488 & $\mathrm{W}(0.124) / \mathrm{N}$ & $\mathrm{W}(0.127) / \mathrm{N}$ & $\mathrm{N}(0.120) / \mathrm{M}$ & $\mathrm{W}(0.127) / \mathrm{W}$ \\
\hline
\end{tabular}

CCUG—strains obtained from Culture Collection University Göteborg, LMG-strains obtained from the Belgian Co-ordinated Collections of Microorganisms, CCM - strains obtained from the Czech Collection of Microorganisms, N—non-adherent, W-weakly adherent, $\mathrm{M}-$ moderately adherent, S—strongly adherent. Value in parentheses represents the actual measured absorbance value.

Table 4. Biofilm formation of Campylobacter strains on plastic/glass material at $42{ }^{\circ} \mathrm{C}$.

\begin{tabular}{|c|c|c|c|c|}
\hline \multirow{2}{*}{ Strains } & \multicolumn{2}{|c|}{ Exposure for $24 \mathrm{~h}$} & \multicolumn{2}{|c|}{ Exposure for $72 \mathrm{~h}$} \\
\hline & Aerobic & Microaerophilic & Aerobic & Microaerophilic \\
\hline Campylobacter jejuni CCM 6214 & $\mathrm{~W}(0.121) / \mathrm{M}$ & $\mathrm{N}(0.115) / \mathrm{M}$ & $\mathrm{N}(0.093) / \mathrm{M}$ & $\mathrm{N}(0.117) / \mathrm{M}$ \\
\hline Campylobacter jejuni NP $2896^{C}$ & $\mathrm{~W}(0.126) / \mathrm{W}$ & $\mathrm{N}(0.119) / \mathrm{W}$ & $\mathrm{N}(0.092) / \mathrm{N}$ & $\mathrm{N}(0.117) / \mathrm{W}$ \\
\hline Campylobacter jejuni NS $3668^{C}$ & $\mathrm{~W}(0.125) / \mathrm{N}$ & $\mathrm{W}(0.127) / \mathrm{N}$ & $\mathrm{N}(0.114) / \mathrm{W}$ & $\mathrm{N}(0.117) / \mathrm{W}$ \\
\hline Campylobacter jejuni NS $3800^{C}$ & $\mathrm{~W}(0.121) / \mathrm{N}$ & $\mathrm{W}(0.127) / \mathrm{W}$ & $\mathrm{N}(0.115) / \mathrm{N}$ & $\mathrm{W}(0.121) / \mathrm{N}$ \\
\hline Campylobacter jejuni NS $4088^{C}$ & $\mathrm{~W}(0.124) / \mathrm{N}$ & $\mathrm{W}(0.127) / \mathrm{N}$ & $\mathrm{N}(0.119) / \mathrm{N}$ & $\mathrm{W}(0.129) / \mathrm{N}$ \\
\hline Campylobacter jejuni NS $4091^{C}$ & $\mathrm{~W}(0.124) / \mathrm{W}$ & $\mathrm{W}(0.125) / \mathrm{N}$ & $\mathrm{N}(0.113) / \mathrm{N}$ & $\mathrm{N}(0.118) / \mathrm{N}$ \\
\hline Campylobacter coli NP $2395^{C}$ & $\mathrm{~W}(0.123) / \mathrm{W}$ & $\mathrm{W}(0.125) / \mathrm{N}$ & $\mathrm{N}(0.118) / \mathrm{N}$ & $\mathrm{W}(0.125) / \mathrm{N}$ \\
\hline Campylobacter coli NS $3803^{C}$ & $\mathrm{~W}(0.121) / \mathrm{W}$ & $\mathrm{N}(0.116) / \mathrm{N}$ & $\mathrm{N}(0.094) / \mathrm{N}$ & $\mathrm{N}(0.116) / \mathrm{N}$ \\
\hline Campylobacter coli NS $4062^{C}$ & $\mathrm{~W}(0.121) / \mathrm{W}$ & $\mathrm{N}(0.115) / \mathrm{N}$ & $\mathrm{N}(0.094) / \mathrm{N}$ & $\mathrm{N}(0.118) / \mathrm{W}$ \\
\hline Campylobacter sp. ONN $366^{C}$ & $\mathrm{~W}(0.132) / \mathrm{W}$ & $\mathrm{W}(0.130) / \mathrm{N}$ & $\mathrm{N}(0.115) / \mathrm{N}$ & $\mathrm{N}(0.118) / \mathrm{W}$ \\
\hline
\end{tabular}

${ }^{C}$ clinical isolate; $C C M$-strains obtained from the Czech Collection of Microorganisms, NP—strains obtained from Pardubice Hospital, NS—strains obtained from Svitavy Hospital, ONN—strains obtained from Náchod Regional Hospital, N—non-adherent, W—weakly adherent, $\mathrm{M}-$ moderately adherent, $\mathrm{S}$ - strongly adherent. Value in parentheses represents the actual measured absorbance value.

\section{Results}

\section{1. $16 S$ rRNA-RFLP and $m$-PCR Identification of Strains}

A total of 51 Arcobacter-like isolates were recovered from food, water samples, or clinical strains. All isolates were successfully identified as A. butzleri or A. cryaerophilus using both mPCR and 16S rRNA-RFLP methods and used for further testing. Two subgroups of $A$. cryaerophilus, $1 \mathrm{~A}$ and $1 \mathrm{~B}$, were further distinguished based on the restriction profiles attained with 16S rRNA-RFLP. Other strains were obtained from collections of microorganisms as properly identified strains. 


\subsection{Biofilm Formation of Arcobacter-like Bacteria under Different Conditions \\ 3.2.1. Biofilm Formation on Plastic (Polystyrene) Material}

The biofilm activity of 59 Arcobacter-like strains was studied under various culture conditions on a polystyrene surface (Tables 1-3). The obtained data reveal that the strains tested differ significantly in the intensity of their biofilm formation. However, an association between strain origin and biofilm activity was not observed at a statistically significant level $(p>0.05)$. The categorization of biofilm activity was influenced by the culture atmosphere in a total of 31 strains (52.5\%) of arcobacters. Of this number, 27 strains (87.1\%) had significantly increased biofilm activity under microaerophilic culture conditions. This can be explained by the fact that arcobacters usually grow under aerobic conditions and microaerophilic conditions subject cells to an increase in stress, to which they may respond by increasing biofilm formation. However, in five strains (8.5\%), increased biofilm formation was observed under aerobic culture conditions. For example, in A. butzleri UPa 39-3, increased biofilm production under aerobic conditions was observed after both 24 and $72 \mathrm{~h}$ of cultivation.

The other monitored factor influencing biofilm formation was exposure time. According to results in Tables 1-3, 18.6/11.9\% of isolates were categorized as non-adherent, $78.0 / 79.7 \%$ as weakly adherent, and $3.4 / 8.5 \%$ were included in the category of moderately adherent under aerobic/microaerophilic conditions after $24 \mathrm{~h}$ of exposure. After $72 \mathrm{~h}$ of exposure, $25.4 / 5.1 \%$ of isolates were categorized as non-adherent, $71.2 / 88.1 \%$ as weakly adherent, and $3.4 / 5.1 \%$ were included in the category of moderately adherent under aerobic/microaerophilic conditions. According to the measured absorbances, the biofilm-forming ability of weakly adherent isolates ranged from 0.121 to 0.240 , moderately adherent isolates exhibited a range of biofilm ability varying from 0.241 to 0.418 . Only one strain $(1.7 \%)$ was rated as strongly adherent under microaerophilic conditions and after $72 \mathrm{~h}$ of exposure.

As was mentioned above, an increased amount of biofilm was observed in some strains after prolonged cultivation. This was most evident in A. defluvii LMG 25694. In this strain, the amount of biofilm detected increased by more than $80.0 \%$ after $72 \mathrm{~h}$ of cultivation under aerobic conditions (see Table 3). The same was observed for the clinical isolate of A. butzleri UPa 39-3 $\left(\mathrm{A}_{595 \_\mathrm{A} 24 \mathrm{~h}}=0.143 \pm 0.006\right.$ and $\left.\mathrm{A}_{595 \_\mathrm{M} 72 \mathrm{~h}}=0.242 \pm 0.017\right)$. However, for example in the A. butzleri UPa 2015/7 strain, a reduced amount of detected biofilm was recorded after $72 \mathrm{~h}$ of cultivation $\left(\mathrm{A}_{595 \_\mathrm{A} 24 \mathrm{~h}}=0.241 \pm 0.011\right.$; and $\left.\mathrm{A}_{595 \_\mathrm{M} 72 \mathrm{~h}}=0.120 \pm 0.003\right)$. However, in most strains approximately the same biofilm production was observed after exposure for 24 and $72 \mathrm{~h}$ under otherwise identical conditions.

\subsubsection{Biofilm Formation on Glass (Borosilicate) Material}

The biofilm activity of 59 Arcobacter-like strains was studied under various culture conditions on borosilicate surfaces (Tables 1-3). The obtained results show significantly different intensities of biofilm produced. However, an association between strain origin and biofilm activity was not observed at a statistically significant level $(p>0.05)$. The vast majority of strains $(58 ; 98.3 \%)$ were confirmed to be capable of adhesion to the borosilicate surface under at least some experimental conditions. The categorization of biofilm activity was influenced by the cultivation atmosphere in a total of 43 strains $(72.9 \%)$ of arcobacters. Of these, 38 strains $(88.4 \%)$ had increased biofilm activity under aerobic cultivation conditions.

According to the results presented in Tables $1-3,27.1 / 32.2 \%$ of isolates were categorized as non-adherent, $30.5 / 45.8 \%$ as weakly adherent, $40.7 / 20.3 \%$ as moderately adherent and $1.7 / 1.7 \%$ were included in the category of strongly adherent under aero$\mathrm{bic} /$ microaerophilic conditions after $24 \mathrm{~h}$ of exposure. After $72 \mathrm{~h}$ of exposure, $8.5 / 23.7 \%$ of isolates were categorized as non-adherent, $20.3 / 49.2 \%$ as weakly adherent, $67.8 / 25.4 \%$ as moderately adherent, and $3.4 / 1.7 \%$ were included in the category of moderately adherent under aerobic/microaerophilic conditions. Higher biofilm production on glass under an aerobic atmosphere was observed in many strains. This applies to strains across the species 
of interest (e.g., A. cryaerophilus 1B UPa 2012/1, A. butzleri UPa 2012/3, A. lanthieri LMG 28517 , etc.).

Further, greater production of arcobacter biofilms on the borosilicate surface was confirmed after a longer cultivation period (Tables 1-3). The strains of A. skirrowii LMG 6621 and A. thereius LMG 24488 did not form a biofilm after $24 \mathrm{~h}$ of cultivation on glass, but after prolonged cultivation the biofilm formation was relatively high. A similar phenomenon was observed with A. butzleri UPa 2015/13, A. butzleri UPa 2015/15, A. butzleri UPa 2015/20, A. cryaerophilus 1B UPa 2014/58A, and A. cryaerophilus 1B UPa 130 (after a longer exposure period, higher biofilm production was observed under both aerobic and microaerophilic conditions).

Our results showed that incubation under an aerobic or microaerophilic atmosphere may influence biofilm formation ability on abiotic surfaces. Atmospheric conditions could therefore be relevant to the food-based transmission of Arcobacter-like bacteria.

\subsection{Biofilm Formation of Campylobacter spp. under Different Conditions}

\subsubsection{Biofilm Formation on Plastic (Polystyrene) Material}

According to Table $4,0.0 / 40.0 \%$ of isolates were categorized as non-adherent, and $100.0 / 60.0 \%$ were included in the category of weakly adherent under aerobic/microaerophilic conditions after $24 \mathrm{~h}$ of exposure. After $72 \mathrm{~h}$ of exposure, $100.0 / 70.0 \%$ of isolates were categorized as non-adherent, and $0.0 / 30.0 \%$ were included in the category of weakly adherent under aerobic/microaerophilic conditions. The measured absorbance-biofilm formation ability of weakly adherent isolates ranged from 0.121 to 0.132 .

In 10 strains $(100.0 \%)$ of campylobacter, the ability to adhere to the polystyrene surface was confirmed under at least some experimental conditions. The categorization of biofilm activity was influenced by the culture atmosphere in 7 strains $(70.0 \%)$. Of these, 4 strains (57.1\%) exhibited increased biofilm activity under aerobic culture conditions.

All campylobacters exhibited adherence under aerobic conditions after $24 \mathrm{~h}$ of exposure. The results obtained probably also indicate in this case that the campylobacters, which usually grow microaerophilically, only exhibit higher biofilm production under unfavorable aerobic cultivation conditions. However, an increase in the amount of biofilm after microaerophilic cultivation was observed in some strains, e.g., in the C. coli NP 2395 strain $\left(\mathrm{A}_{595 \_\mathrm{M} 72 \mathrm{~h}}=0.125 \pm 0.007\right.$; and $\left.\mathrm{A}_{595 \_\mathrm{A} 72 \mathrm{~h}}=0.118 \pm 0.003\right)$.

According to our results, no biofilm formation was observed in most strains $(70.0 \%)$ after $72 \mathrm{~h}$ of exposure. The biofilm was only detected after $72 \mathrm{~h}$ of exposure in the $C$. jejuni NS 3800, C. jejuni NS 4088, and C. coli NP 2395 strains. The cultivation time is probably so long that the biofilm structure is already dying and gradually peeling off the surface of the material.

\subsubsection{Biofilm Formation on Glass (Borosilicate) Material}

Most Campylobacter strains (90.0\%) were confirmed to be able to adhere to the borosilicate surface at least under some experimental conditions. The categorization of biofilm activity was influenced by the cultivation atmosphere in seven strains $(70.0 \%)$ of campylobacters. Of these, four strains (57.1\%) exhibited increased adherence under microaerophilic conditions of cultivation. According to Table 4, 30.0/70.0\% of isolates were categorized as non-adherent, and $60.0 / 20.0 \%$ were included in the category of weakly adherent under aerobic/microaerophilic conditions after $24 \mathrm{~h}$ of exposure. After $72 \mathrm{~h}$ of exposure, $80.0 / 50.0 \%$ of isolates were categorized as non-adherent, and 10.0/40.0\% were included in the category of weakly adherent under aerobic/microaerophilic conditions. Overall, the highest biofilm production on the borosilicate surface was observed in the C. jejuni CCM 6214 strain under all experimental conditions.

After $24 \mathrm{~h}$ of aerobic cultivation, only weak biofilm formation was observed in campylobacters; in C. jejuni NS3668, C. jejuni NS3800 and C. jejuni NS4088, no biofilm formation was detected at all under the given conditions. After prolonged cultivation $(72 \mathrm{~h})$ in an 
aerobic environment, $80.0 \%$ of strains no longer exhibited biofilm formation (with the exception of C. jejuni CCM 6214 and C. jejuni NS 3668).

\subsection{Biofilm Formation on Food Processing Materials under Various Conditions}

Based on previous testing, Arcobacter-like and Campylobacter strains that showed moderate to strong biofilm formation activity were selected for further monitoring of biofilm formation on materials used in the food industry or in food processing (glass, plastic, stainless steel). The results of this testing are presented on Figure 1. The results show that the highest biofilm activity was observed in the case of A. defluvii LMG 25694. Increasing biofilm activity was also observed in this strain with increasing culture temperature. The highest biofilm formation in the case of this strain was observed after cultivation at $30{ }^{\circ} \mathrm{C}$ on stainless steel. The lowest amount of biofilm was observed in the strain A. butzleri UPa $2013 / 3$ after cultivation at $5{ }^{\circ} \mathrm{C}$ on stainless steel, but at higher cultivation temperatures the detected amount of biofilm in this strain was highest on stainless steel. Relatively balanced biofilm formation was observed in Campylobacter jejuni NP 2896 strain. There were no significant differences in the formation of the amount of biofilm produced on individual materials in this strain. Nevertheless, it can be stated that lower biofilm formation was observed at the optimal culture temperature of Campylobacter $\left(42{ }^{\circ} \mathrm{C}\right)$, compared to lower temperatures.

A

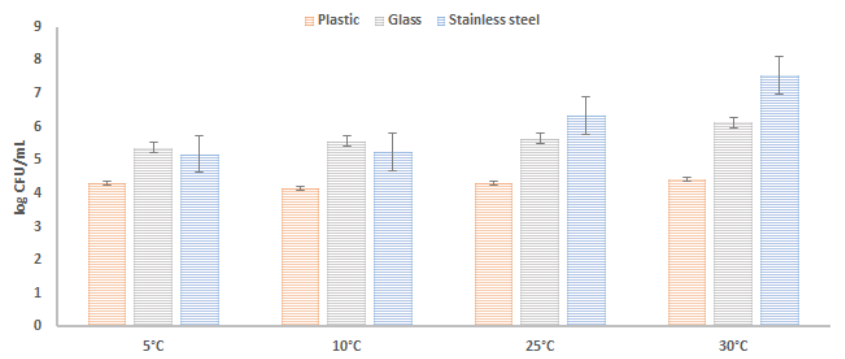

C

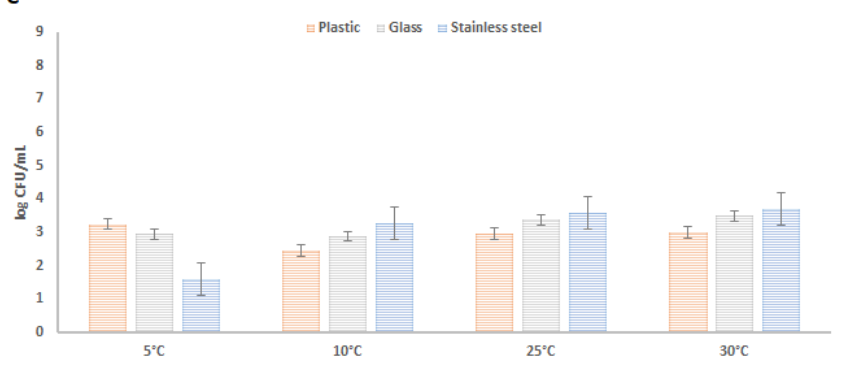

B

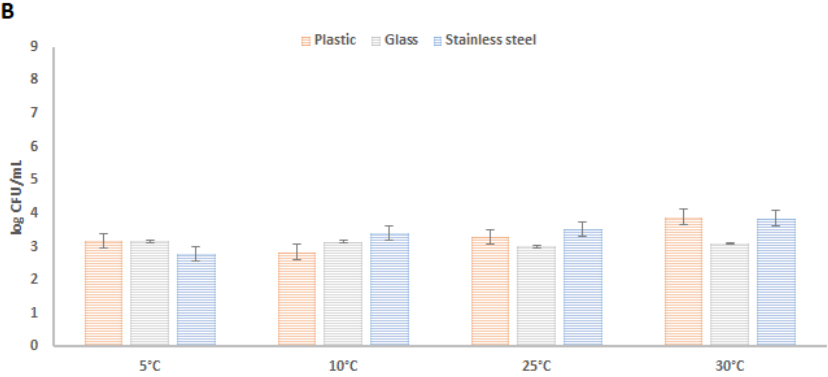

D

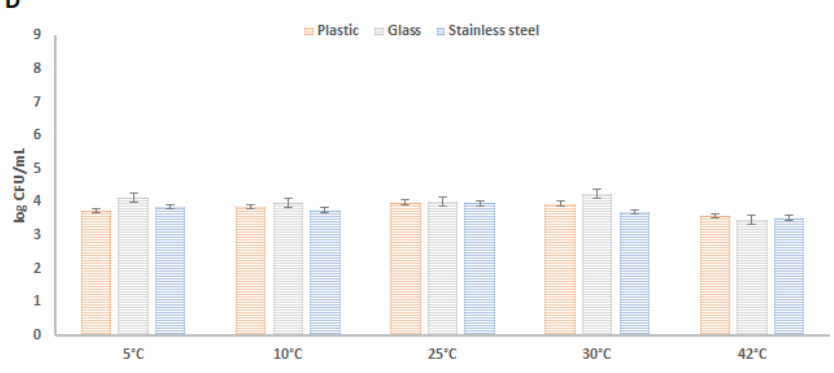

Figure 1. Biofilm formation of Arcobacter-like ((A)—A. defluvii LMG 25694; (B)—A. cryaerophilus 1B UPa 2012/1; (C)—A. butzleri UPa 2013/3) and Campylobacter ((D)—C. jejuni NP 2896) strains on food processing materials at various temperatures and aerobic conditions.

\section{Discussion}

The correct identification of microbial isolates is the basis for further experimental work. Several molecular methods have been designed for Arcobacter identification at the species level. The most globally used method is mPCR developed by Houf et al., although this method produces misidentification of some recently described species. However, the PCR protocol described by Douidah et al. is able to distinguish more species and provide more reliable results [34] and this protocol is often used to identify arcobacters isolated from many samples, including clinical isolates [25]. According to our results, this mPCR method is suitable for the identification of arcobacters, especially in view of the lower possibility of confusion of identified species. However, $16 \mathrm{~S}$ rRNA-RFLP is able to help with identification of arcobacters, including recently described ones. This method is reproducible, reliable, simple, fast, and reasonably inexpensive. The technique is highly 
applicable for investigations of the prevalence of Arcobacter in a variety of food products, water, or other samples [29]. Based on the results of identification using 16S rRNA-RFLP, it was also possible to distinguish the $A$. cryaerophilus isolates included in this study into subgroups $1 \mathrm{~A}$ and $1 \mathrm{~B}$.

The genera Campylobacter and Arcobacter have become increasingly important in recent years because their members have been considered emergent enteropathogens and potential zoonotic agents [34]. Arcobacter-like bacteria, mostly A. butzleri, are frequently isolated from products of animal origin with the highest prevalence occurring in poultry products [19]. However, the occurrence of these bacteria might be underestimated due to no strict methodology for the isolation and identification of these bacteria. Biofilm formation ability is among the factors influencing bacterial virulence, antibiotic resistance, etc. The biofilm formation of arcobacters and campylobacters has been previously described, for example on stainless steel, copper, glass, and plastic surfaces [17,19,35-40]. However, arcobacters are generally described in the literature as weak biofilm producers [38]. Similarly, many studies indicate that campylobacters are not strong biofilm producers [17,39-41], which is consistent with the results of our study. According to the obtained results, it can be stated that the Campylobacter species exhibited slightly higher biofilm production in their natural microaerophilic environment, which has also been reported elsewhere [17].

In some studies, higher biofilm activity has been reported in strains isolated from meat [38], which was not shown to be statistically significant $(p>0.05)$ in our results. To our knowledge, relatively recently taxonomically incorporated, and hence substantially less described, Arcobacter-like species have not been included in any similar studies yet. In this respect, positive biofilm formation on polystyrene surfaces has been found, for example, in A. skirrowii LMG 6621, A. thereius LMG 24488, A. lanthieri LMG 28517 and in particular A. defluvii LMG 25694, in which the highest biofilm formation was found under all experimental conditions. However, it is apparent that biofilm formation is a variable capability and, in some circumstances, a strain may not produce a biofilm at all, even though it has been previously described as biofilm-positive strain. Our results show that the biofilm formation of strains isolated from a real environment does not differ significantly $(p>0.05)$ from the collection type strains, as evidenced also in a previous study [42]. However, another previous study reported that Arcobacter strains isolated from the environment exhibited a greater ability to form biofilms compared to collection strains [19].

Significant external factors such as environmental conditions or surface properties also influence biofilm formation. As has been previously mentioned, it is not generally possible to define which culture conditions are or are not suitable for biofilm formation $[37,38]$. The availability of nutrients or the composition of the culture medium used also has a significant influence on biofilm formation. For example, TSB medium [43] or BHI broth [44] are often used for testing. In contrast, Mueller-Hinton broth is not nutritionally well suited for these purposes [38].

The ability to adhere to the polystyrene surface of the microtiter plates was confirmed in at least some experimental conditions in all $59(100.0 \%)$ of the studied Arcobacter-like strains. Some studies report the biofilm formation ability of a high number of Arcobacter strains [19,35]; in other studies, this was confirmed in only $21.4 \%$ of the studied strains [38]. Obviously, the type of material significantly affects the adherence and biofilm formation of arcobacters [37,38]. According to our results, A. butzleri UPa 2015/12 strain was rated as non-adherent on all materials and under all conditions. However, adherence-capable strains were generally rated as stronger biofilm producers than the strains that adhered to plastic surfaces only (see Tables 1-3).

It has been confirmed in the past that biofilm activity is influenced by the culture atmosphere [19,38]. An aerobic environment may promote the adhesion properties of some Arcobacter-like strains, while other strains are stimulated by microaerophilic conditions [19]. Earlier studies also reached similar conclusions in the case of Campylobacter, suggesting that the unnatural aerobic atmosphere promotes the formation of a biofilm structure $[17,27]$. 
Based on our results, higher biofilm production under an aerobic atmosphere was recorded; however, some strains shown increase in biofilm formation in microaerophilic atmospheres.

Exposure time has a significant effect on biofilm formation. However, it cannot be stated that a longer cultivation time always means a higher amount of detected biofilm on a given material. This is probably related to different cell tolerance to environmental influences, the susceptibility of the biofilm layer to peeling, etc. It has been reported in the past that $A$. butzleri and $A$. cryaerophilus produced the most biofilm after $24 \mathrm{~h}$ of cultivation and then there was no significant change in the amount of biofilm detected [35] that is consistent with our results. However, some authors also mention that the amount of produced biofilm increases with increasing cultivation time [35]. In an earlier study, it was confirmed that campylobacters are still able to form a biofilm after $48 \mathrm{~h}$ of cultivation [37]. For many strains included in our study, no biofilm formation was observed after $72 \mathrm{~h}$ of exposure, which may be related to the peeling of the formed "integral" layer of biofilm on the given material.

When monitoring the biofilm formation on coupons made of various materials, it is a simulation of more realistic conditions that we encounter in food processing. Stainless steel or plastic material is widely used in the food industry because it exhibits some of the most suitable characteristics of the construction materials used for food equipment [38]. The ability of Arcobacter and Campylobacter spp. to adhere to these inert surfaces in the form of biofilms has previously been described [35,41]. The first study describing the adhesion of $A$. butzleri to stainless steel was published in 2002 [45]. Subsequently, another study confirmed the formation of a biofilm on stainless steel in just $14.3 \%$ of studied strains of A. butzleri [35]. Therefore, the findings of the current studies provide confirmation of the ability of $A$. butzleri to adhere to extensively used materials in the food industry $[37,38]$. However, most studies present the results of arcobacters biofilm formation at $30{ }^{\circ} \mathrm{C}$. The results of our study show that arcobacters are capable of relatively large biofilm production, even at lower temperatures such as refrigeration temperatures. Most studies on C. jejuni biofilms have thus far been carried out at $37^{\circ} \mathrm{C}$ [46]. However, Dykes et al. found that C. jejuni grown as planktonic cells and as biofilm cells survived longer at lower temperatures $\left(4^{\circ} \mathrm{C}\right.$ and $\left.10^{\circ} \mathrm{C}\right)$ in comparison with higher temperatures $\left(25^{\circ} \mathrm{C}\right.$ and $\left.37^{\circ} \mathrm{C}\right)$ under stress conditions [47]. While most studies on biofilm formation by $C$. jejuni have been carried out under microaerobic conditions, some of them have been undertaken to examine biofilm formation by C. jejuni under aerobic conditions. The biofilm formation of C. jejuni is often evaluated to be more intense under aerobic conditions [27]. This can be explained by higher biofilm production in response to oxidative stress. Significant biofilm formation of Campylobacter spp. may be of concern, namely even at low temperatures and for a relatively short time.

Even if consumers expect to acquire healthy and safe food products, pathogenic bacteria can be present in a variety of food products. The persistence of pathogenic bacteria in food processing environments leads to food-associated infections. Foodborne illnesses are a growing public health problem worldwide, and their prevention is the main objective of food safety. In this respect, the prevention of cross-contamination is a key factor. However, further investigations are needed to verify the effect of biofilm formation in the spread of pathogens in the food industry.

\section{Conclusions}

This study provides information on the biofilm activity of many Arcobacter-like strains and Campylobacter spp. isolated from the environment, food or clinical samples collected within the Czech Republic. The results of the study confirm the ability to form a biofilm in almost all the strains involved in this study. However, the resulting biofilm formation is variable and dependent on many environmental factors, especially the atmosphere and the length of the cultivation. Higher adhesion was observed in conditions less favorable for the survival of planktonic forms of these bacteria-under microaerophilic or aerobic conditions in the case of Arcobacter spp. or Campylobacter spp., respectively. 
As far as we know, this is the first study describing biofilm formation in such a large set of strains, including representatives of relatively recently described Arcobacter-like species. The study also provides an important insight into the ability of many clinical isolates of Arcobacter and Campylobacter to adhere to surfaces and form a biofilm. According to our results, the selected strains were able to form a biofilm on food processing materials, even more so at lower temperatures for C. jejuni NP 2896.

These data contribute to the understanding of the survival and persistence of these bacteria in their environment, their potential virulence, and relevance as potential pathogens in the food chain or healthcare. It is important to monitor the biofilm formation capability of these bacteria, as they are responsible for a significant percentage of human alimentary infections. Ideally, conditions should be set to prevent the formation of biofilm structures. Subsequent studies of the options for influencing the formation of a biofilm would have great benefits in both the food industry and healthcare.

Author Contributions: Conceptualization, D.Š.; methodology, D.Š. and K.K.; formal analysis, D.Š. and L.Š.; investigation, S.S., D.Š., K.Š., L.H., L.Š. and K.K.; writing—original draft preparation, D.Š.; writing - review and editing, D.Š. and K.Š.; supervision, D.Š.; project administration, D.Š.; funding acquisition, D.Š. All authors have read and agreed to the published version of the manuscript.

Funding: This research received no external funding.

Acknowledgments: The authors thank Barbora Hurdálková from Náchod Hospital and Jana Vobejdová from Litomyšl Hospital for kindly providing strains of clinical isolates of Aliarcobacter butzleri and Campylobacter jejuni.

Conflicts of Interest: The authors declare no conflict of interest. The funders had no role in the design of the study; in the collection, analyses, or interpretation of data; in the writing of the manuscript; or in the decision to publish the results.

\section{References}

1. Donlan, R.M.; Costerton, J.W. Biofilms: Survival Mechanisms of Clinically Relevant Microorganisms. Clin. Microbiol. Rev. 2002, 15, 167-193. [CrossRef]

2. Sutherland, I.W. Biofilm exopolysaccharides: A strong and sticky framework. Microbiology 2001, 147, 3-9. [CrossRef]

3. Mah, T.-F.C.; O'Toole, G.A. Mechanisms of biofilm resistance to antimicrobial agents. Trends Microbiol. 2001, 9, 34-39. [CrossRef]

4. Mulcahy, H.; Charron-Mazenod, L.; Lewenza, S. Extracellular DNA Chelates Cations and Induces Antibiotic Resistance in Pseudomonas aeruginosa Biofilms. PLoS Pathog. 2008, 4, e1000213. [CrossRef]

5. Kwiecińska-Piróg, J.; Przekwas, J.; Majkut, M.; Skowron, K.; Gospodarek-Komkowska, E. Biofilm Formation Reducing Properties of Manuka Honey and Propolis in Proteus mirabilis Rods Isolated from Chronic Wounds. Microorganisms 2020, 8, 1823. [CrossRef]

6. Šilha, D.; Švarcová, K.; Bajer, T.; Královec, K.; Tesařová, E.; Moučková, K.; Pejchalová, M.; Bajerová, P. Chemical Composition of Natural Hydrolates and Their Antimicrobial Activity on Arcobacter-Like Cells in Comparison with Other Microorganisms. Molecules 2020, 25, 5654. [CrossRef]

7. Aparna, M.S.; Yadav, S. Biofilms: Microbes and disease. Braz. J. Infect. Dis. 2008, 12, 526-530. [CrossRef] [PubMed]

8. Jamal, M.; Ahmad, W.; Andleeb, S.; Jalil, F.; Imran, M.; Nawaz, M.A.; Hussain, T.; Ali, M.; Rafiq, M.; Kamil, M.A. Bacterial biofilm and associated infections. J. Chin. Med. Assoc. 2018, 81, 7-11. [CrossRef] [PubMed]

9. Zhao, X.; Yu, Z.; Ding, T. Quorum-Sensing Regulation of Antimicrobial Resistance in Bacteria. Microorganisms 2020, 8, 425. [CrossRef]

10. Naves, P.L.F.; Del Prado, G.; Huelves, L.; Gracia, M.; Ruiz, V.; Blanco, J.; Rodríguez-Cerrato, V.; Ponte, M.; Soriano, F. Measurement of biofilm formation by clinical isolates of Escherichia coli method-dependent. J. Appl. Microbiol. 2008, 105, 585-590. [CrossRef] [PubMed]

11. Silva, V.; Peirone, C.; Amaral, J.S.; Capita, R.; Alonso-Calleja, C.; Marques-Magallanes, J.A.; Martins, Â.; Carvalho, Á.; Maltez, L.; Pereira, J.E.; et al. High Efficacy of Ozonated Oils on the Removal of Biofilms Produced by Methicillin-Resistant Staphylococcus aureus (MRSA) from Infected Diabetic Foot Ulcers. Molecules 2020, 25, 3601. [CrossRef] [PubMed]

12. Srey, S.; Jahid, I.K.; Ha, S.-D. Biofilm formation in food industries: A food safety concern. Food Control 2013, 31, 572-585. [CrossRef]

13. Costerton, J.W.; Stewart, P.; Greenberg, E. Bacterial Biofilms: A Common Cause of Persistent Infections. Science 1999, 21, 1318-1322. [CrossRef] [PubMed]

14. Candon, H.L.; Allan, B.J.; Fraley, C.D.; Gaynor, E.C. Polyphosphate Kinase 1 Is a Pathogenesis Determinant in Campylobacter jejuni. J. Bacteriol. 2007, 189, 8099-8108. [CrossRef]

15. Christensen, G.D.; Simpson, W.A.; Bisno, A.L.; Beachey, E.H. Adherence of slime-producting strains of Staphylococcus-epidermidis to smooth surfaces. Infect. Immun. 1982, 37, 318-326. [CrossRef] 
16. Niu, C.; Gilbert, E.S. Colorimetric Method for Identifying Plant Essential Oil Components That Affect Biofilm Formation and Structure. Appl. Environ. Microbiol. 2004, 70, 6951-6956. [CrossRef]

17. Guntheriv, N.; Chen, C. The biofilm forming potential of bacterial species in the genus Campylobacter. Food Microbiol. 2009, 26, 44-51. [CrossRef]

18. Mizan, F.R.; Jahid, I.K.; Ha, S.-D. Microbial biofilms in seafood: A food-hygiene challenge. Food Microbiol. $2015,49,41-55$. [CrossRef]

19. Ferreira, S.; Fraqueza, M.J.; Queiroz, J.; Domingues, F.; Oleastro, M. Genetic diversity, antibiotic resistance and biofilm-forming ability of Arcobacter butzleri isolated from poultry and environment from a Portuguese slaughterhouse. Int. J. Food Microbiol. 2013, 162, 82-88. [CrossRef]

20. Silha, D.; Moravkova, K.; Skodova, G.; Vytrasova, J. Viability and biofilm formation of Arcobacter spp. at various processing temperatures. J. Food Nutr. Res. 2019, 58, 208-213.

21. Šilha, D.; Šilhová-Hrušková, L.; Vytřasová, J. Modified isolation method of Arcobacter spp. from different environmental and food samples. Folia Microbiol. 2015, 60, 515-521. [CrossRef] [PubMed]

22. Elmali, M.; CAN, H.Y. Occurence and antimicrobial resistance of Arcobacter species in food and slaughterhouse samples. Food Sci. Technol. 2017, 37, 280-285. [CrossRef]

23. Waite, D.; Vanwonterghem, I.; Rinke, C.; Parks, D.H.; Zhang, Y.; Takai, K.; Sievert, S.; Simon, J.; Campbell, B.J.; Hanson, T.; et al. Comparative Genomic Analysis of the Class Epsilonproteobacteria and Proposed Reclassification to Epsilonbacteraeota (phyl. nov.). Front. Microbiol. 2017, 8, 1-19. [CrossRef] [PubMed]

24. Pérez-Cataluña, A.; Salas-Massó, N.; Diéguez, A.L.; Balboa, S.; Lema, A.; Romalde, J.L.; Figueras, M.J. Revisiting the Taxonomy of the Genus Arcobacter: Getting Order from the Chaos. Front. Microbiol. 2018, 9, 2077. [CrossRef]

25. Abeele, A.-M.V.D.; Vogelaers, D.; Van Hende, J.; Houf, K. Prevalence of Arcobacter Species among Humans, Belgium, $2008-2013$. Emerg. Infect. Dis. 2014, 20, 364-383. [CrossRef]

26. Ferreira, S.; Queiroz, J.; Oleastro, M.; Domingues, F. Insights in the pathogenesis and resistance of Arcobacter: A review. Crit. Rev. Microbiol. 2016, 42, 1-20. [CrossRef]

27. Reuter, M.; Mallett, A.; Pearson, B.; van Vliet, A.H.M. Biofilm Formation by Campylobacter jejuni Is Increased under Aerobic Conditions. Appl. Environ. Microbiol. 2010, 76, 2122-2128. [CrossRef]

28. Douidah, L.; De Zutter, L.; Vandamme, P.; Houf, K. Identification of five human and mammal associated Arcobacter species by a novel multiplex-PCR assay. J. Microbiol. Methods 2010, 80, 281-286. [CrossRef]

29. Figueras, M.J.; Levican, A.; Collado, L. Updated 16S rRNA-RFLP method for the identification of all currently characterised Arcobacter spp. BMC Microbiol. 2012, 12, 292. [CrossRef]

30. Marshall, S.M.; Melito, P.L.; Woodward, D.L.; Johnson, W.M.; Rodgers, F.G.; Mulvey, M.R. Rapid Identification of Campylobacter, Arcobacter, and Helicobacter Isolates by PCR-Restriction Fragment Length Polymorphism Analysis of the 16S rRNA Gene. J. Clin. Microbiol. 1999, 37, 4158-4160. [CrossRef]

31. Christensen, G.D.; Simpson, W.A.; Younger, J.J.; Baddour, L.M.; Barrett, F.F.; Melton, D.M.; Beachey, E.H. Adherence of coagulasenegative staphylococci to plastic tissue culture plates: A quantitative model for the adherence of staphylococci to medical devices. J. Clin. Microbiol. 1985, 22, 996-1006. [CrossRef] [PubMed]

32. Stepanović, S.; Vuković, D.; Dakić, I.; Savić, B.; Švabić-Vlahović, M. A modified microtiter-plate test for quantification of staphylococcal biofilm formation. J. Microbiol. Methods 2000, 40, 175-179. [CrossRef]

33. Blackman, I.C.; Frank, J.F. Growth of Listeria monocytogenes as a Biofilm on Various Food-Processing Surfaces. J. Food Prot. 1996, 59, 827-831. [CrossRef] [PubMed]

34. Collado, L.; Figueras, M.J. Taxonomy, Epidemiology, and Clinical Relevance of the Genus Arcobacter. Clin. Microbiol. Rev. 2011, 24, 174-192. [CrossRef]

35. Kjeldgaard, J.; Jørgensen, K.; Ingmer, H. Growth and survival at chiller temperatures of Arcobacter butzleri. Int. J. Food Microbiol. 2009, 131, 256-259. [CrossRef]

36. Silha, D.; Hruskova, L.; Brozkova, I.; Motkova, P.; Vytrasova, J. Survival of selected bacteria from the genus Arcobacter on various metallic surfaces. J. Food Nutr. Res. 2014, 53, 217-223.

37. Šilhová-Hrušková, L.; Mot'ková, P.; Šilha, D.; Vytřasová, J. [Detection of biofilm formation by selected pathogens relevant to the food industry]. Epidemiol. Mikrobiol. Imunol. 2015, 64, 169-175.

38. Girbau, C.; Martinez-Malaxetxebarria, I.; Muruaga, G.; Carmona, S.; Alonso, R.; Fernandez-Astorga, A. Study of Biofilm Formation Ability of Foodborne Arcobacter butzleri under Different Conditions. J. Food Prot. 2017, 80, 758-762. [CrossRef] [PubMed]

39. Joshua, G.W.P.; Guthrie-Irons, C.; Karlyshev, A.V.; Wren, B.W. Biofilm formation in Campylobacter jejuni. Microbiology 2006, 152, 387-396. [CrossRef]

40. Reeser, R.J.; Medler, R.T.; Billington, S.J.; Jost, B.H.; Joens, L.A. Characterization of Campylobacter jejuni Biofilms under Defined Growth Conditions. Appl. Environ. Microbiol. 2007, 73, 1908-1913. [CrossRef]

41. Teh, K.H.; Flint, S.; French, N. Biofilm formation by Campylobacter jejuni in controlled mixed-microbial populations. Int. J. Food Microbiol. 2010, 143, 118-124. [CrossRef]

42. Hrušková, L.; Mot'Ková, P.; Vytřasová, J. Multiplex polymerase chain reaction using ethidium monoazide and propidium monoazide for distinguishing viable and dead cells of arcobacters in biofilm. Can. J. Microbiol. 2013, 59, 797-802. [CrossRef] 
43. Prakash, P.; Singh, A.; Achra, A.; Singh, G.P.; DAS, A.; Singh, R.K. Standardization and classification of In vitro biofilm formation by clinical isolates of Staphylococcus aureus. J. Glob. Infect. Dis. 2017, 9, 93-101. [CrossRef]

44. Knobloch, J.K.-M.; Horstkotte, M.A.; Rohde, H.; Mack, D. Evaluation of different detection methods of biofilm formation in Staphylococcus aureus. Med. Microbiol. Immunol. 2002, 191, 101-106. [CrossRef]

45. Assanta, M.A.; Roy, D.; Lemay, M.-J.; Montpetit, D. Attachment of Arcobacter butzleri, a New Waterborne Pathogen, to Water Distribution Pipe Surfaces. J. Food Prot. 2002, 65, 1240-1247. [CrossRef] [PubMed]

46. Teh, A.H.T.; Lee, S.M.; Dykes, G.A. Does Campylobacter jejuni Form Biofilms in Food-Related Environments? Appl. Environ. Microbiol. 2014, 80, 5154-5160. [CrossRef] [PubMed]

47. Dykes, G.; Sampathkumar, B.; Korber, D. Planktonic or biofilm growth affects survival, hydrophobicity and protein expression patterns of a pathogenic Campylobacter jejuni strain. Int. J. Food Microbiol. 2003, 89, 1-10. [CrossRef] 